\title{
Myxoedematous polyneuropathy: a light and electron microscopic study of the peripheral nerve and muscle
}

\author{
T. ShIRABE, S. TAWARA, A. TERAO, AND S. ARAKI \\ From the Divisions of Neuropathology and Neurology, \\ Kawasaki Medical College, Kurashiki 701-01, Japan
}

SYNOPSIS Histopathological findings of biopsied peripheral nerve and muscle were studied in a case with myxoedematous polyneuropathy. The most striking findings in the sural nerve were segmental demyelination and onion bulb formation with scanty mucinous deposits in addition to marked loss of large myelinated nerve fibres. The peroneus brevis muscle revealed the association of neuropathic and myopathic changes. It is suggested that myxoedematous polyneuropathy might be intrinsic neuropathy due to metabolic disorder of Schwann cells related to hypothyroidism, resulting in segmental demyelination, not merely compressive neuropathy due to mucinous deposits in the peripheral nerves.

It is well known that myxoedema is associated with many neuropsychiatric manifestations such as headache, forgetfulness, blurring of vision, tinnitus, deafness, ataxia, mental retardation, psychoses, convulsions, and coma (Nickel et al., 1958; Sanders, 1962; Watanakunakorn et al., 1965). In some patients with this disorder, neuropathy and myopathy may also be the major and presenting symptoms (Crevasse and Logue, 1959; Collins et al., 1970). An important feature of neuropathy in myxoedema is acroparaesthesia in hands or carpal tunnel syndrome (Murray and Simpson, 1958; Purnell et al., 1961). Such myxoedematous neuropathy has been usually assumed to be compressive neuropathy due to mucinous deposits in the peripheral nerves and their surrounding tissue. A few examples with myxoedema, however, show diffuse polyneuropathy. And yet, to the best of our knowledge, there are few records of a pertinent histological examination of the peripheral nerves in persons with diffuse polyneuropathy associated with myxoedema, which is inadequately explained solely as compressive neuropathy.

The purpose of this paper is to describe some additional histopathological findings of biopsied sural nerve and peroneus brevis muscle in a case (Accepted 27 August 1974.) of diffuse polyneuropathy occurring in a woman with myxoedema and to discuss a possible pathogenesis of this type of polyneuropathy.

\section{CASE REPORT}

A 67 year old woman was admitted to the Kawasaki Medical College Hospital because of numbness in the extremities on 5 September 1973.

Ten years previously oedema had developed transiently in her face, hands, and legs. At that time she had been found in another hospital to have anaemia and heart failure, but no other details were available. Since then, she had been in good health until early in July 1973, when her legs, and subsequently her face and hands, began gradually to swell up. During the same time she experienced difficulty in climbing stairs because of a feeling of heaviness in her legs. She showed a tendency to constipation. Soon she noticed numbness in her hands and feet. In August hearing impairment developed bilaterally. She had a heavy feeling in her head all day. Numbness in the extremities progressed further. She became awkward in skilled finger movements. Swelling in the submaxillary area was noted for a few weeks before admission. She had not been troubled with cramp or stiffness in the muscles. Her family history was unremarkable.

Physical examination on admission revealed a woman of dull appearance, poorly nourished and with a puffy and pale face. Temperature was $36.6^{\circ} \mathrm{C}$. 
The pulse rate was 56 per minute and regular. Blood pressure was $138 / 70 \mathrm{mmHg}$. The eyelids were oedematous. The skin was coarse, dry, and wrinkled. Submaxillary glands were enlarged. The heart, lungs, and abdomen appeared normal. Oedema of nonpitting type was present in her hands and feet.

Neurologically, she was alert and well oriented although she was slow in movements. There was bilateral hearing impairment. Gross power in the extremities was slightly weak in the periphery. No evident atrophy or hypertrophy was observed. Mounding phenomenon was elicited easily in the whole skeletal musculature. Deep tendon reflexes were sluggish in the upper extremities and absent in the lower ones. There was no Babinski sign. Sensation to touch, pain, temperature, and vibration was slightly to moderately diminished in the right hand, below the left elbow, and below both knees, more marked on the left. Joint position sense was slightly reduced in the toes. Standing was possible with difficulty. The Romberg test was equivocal; she walked with an unsteady gait. She had a tendency to constipation and difficulty in urination.

Routine urinalysis on admission was normal. Haematocrit was $29 \%$; erythrocyte count was 2.71 millions $/ \mathrm{mm}^{3}$; leucocyte count was $3100 / \mathrm{mm}^{3}$ with a normal differential count. Erythrocyte sedimentation rate was $18 \mathrm{~mm}$ in one hour. Serum protein concentration was $62 \mathrm{~g} / \mathrm{l}$, with $62.9 \%$ of albumin and $37.1 \%$ of globulin. Beta-lipoprotein level was $1180 \mathrm{mg} / \mathrm{dl}$ (normal: 200 to $500 \mathrm{mg} / \mathrm{dl}$ ). Blood sugar was $68 \mathrm{mg}$, and serum cholesterol was $259 \mathrm{mg} / \mathrm{dl}$. Serum electrolytes, serum creatine and creatinine, transaminase, and urea nitrogen were all within normal limits. Serum creatine phosphokinase was 42 units (normal: less than 12 units), lactic dehydrogenase was 1170 units (normal: 100 to 350 units), and aldolase was 39 units (normal: 3 to 8 units). An electrocardiogram showed sinus bradycardia with low voltage. There was no heart enlargement in a chest radiograph.

Basal metabolic rate was lowered at $-8 \% ; \mathrm{I}^{131}$ uptake rate was 1.6\% (normal: 10 to $40 \%$ ); triosorb test was $19 \%$ (normal: 25 to $35 \%$ ); tetrasorb test was $0.7 \mu \mathrm{g} / \mathrm{dl}$ (normal: 6 to $11 \mu \mathrm{g} / \mathrm{dl}$ ); serum protein bound iodine was $1.5 \mu \mathrm{g} / \mathrm{dl}$ (normal: 4 to $8 \mu \mathrm{g} / \mathrm{dl}$ ); bioassay titre of thyroid stimulating hormone was $104 \mu \mathrm{u} / \mathrm{ml}$ (normal: $5 \mu \mathrm{u} / \mathrm{ml}$ ).

Spinal fluid was clear and colourless, demonstrating an opening pressure of $150 \mathrm{~mm}$ water; cell count was less than one cell per $\mathrm{mm}^{3}$; protein, 72 $\mathrm{mg} / \mathrm{dl}$; sugar, $58 \mathrm{mg} / \mathrm{dl}$; chloride, $125 \mathrm{~mol} / 1$. Electroencephalography revealed an irregular pattern consisting of slow waves of 7-8 Hz and low to moderate voltage, combined with occasional fast waves. Electromyographic examination showed normal insertion activity and no spontaneous activity. The voluntary activity revealed slightly reduced motor unit potentials in all the muscles examined. Motor nerve conduction velocity was $59.0 \mathrm{~m} / \mathrm{s}$ in the right ulnar nerve (normal: greater than $49.0 \mathrm{~m} / \mathrm{s}$ ). Sensory nerve conduction velocity was $23.9 \mathrm{~m} / \mathrm{s}$ from the finger tip to the wrist (normal: 39.8 to $51.0 \mathrm{~m} / \mathrm{s}$ ) and $43.2 \mathrm{~m} / \mathrm{s}$ from the wrist to the elbow (normal: 62.0 to $72.4 \mathrm{~m} / \mathrm{s}$ ) in the right median nerve.

The patient was given dessicated thyroid, $25 \mathrm{mg}$ a day from 13 September, which was gradually increased to $50 \mathrm{mg}$ a day in a month. In October thyroid function was restored to the normal range. Her neurological condition began to improve in two weeks and showed marked improvement after two months of therapy. Muscle power returned to normal. Sensory impairment was limited only to the lower extremities. Superficial and deep sensation was slightly decreased below the knee level on both sides. Repeated sensory conduction times returned to the lower limit of normal. Her gait became almost normal. She is now under control as an outpatient.

\section{METHODS}

Specimens were obtained from the left sural nerve and peroneus brevis muscle on 13 September 1973.

Biopsied sural nerve was divided into two por-

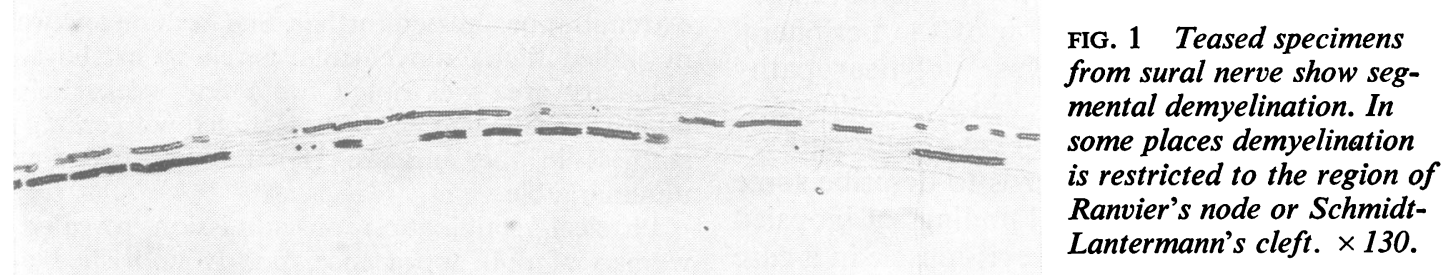

FIG. 1 Teased specimens from sural nerve show segmental demyelination. In is restricted to the region of Ranvier's node or SchmidtLantermann's cleft. $\times 130$. 


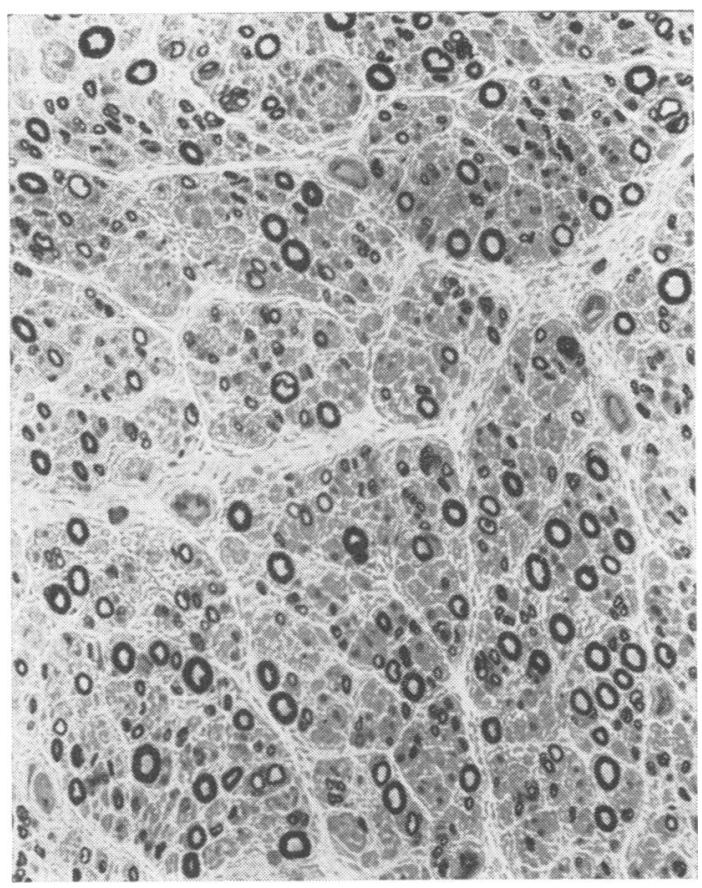

FIG. 2 Sural nerve biopsy specimen. Myelinated nerve fibres, especially large ones, are reduced in number. Toluidine blue, $\times 260$.

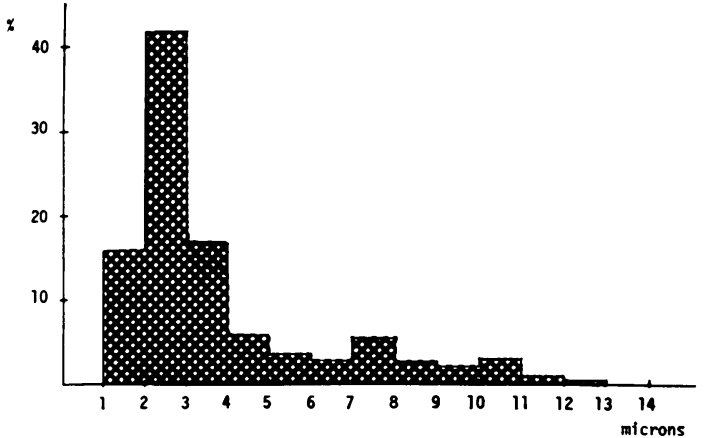

FIG. 3 Histogram of the myelinated nerve fibre diameter. It demonstrates decrease of large fibres, and loss of normal bimodal distribution.

tions. The first segment was fixed in $10 \%$ formalin for routine histological study. Part of the segment was embedded in paraffin wax and stained with haematoxylin and eosin, luxol fast blue of KlüverBarrera, silver stain of Bodian, Mallory-azan, periodic acid-Schiff, mucicarmine, toluidine blue, and alcian blue. Another part, after fixation in $10 \%$ formalin, was subsequently stained in $2 \%$ osmic acid for 24 hours, macerated in glycerol, and teased apart to isolate single nerve fibres under a dissecting

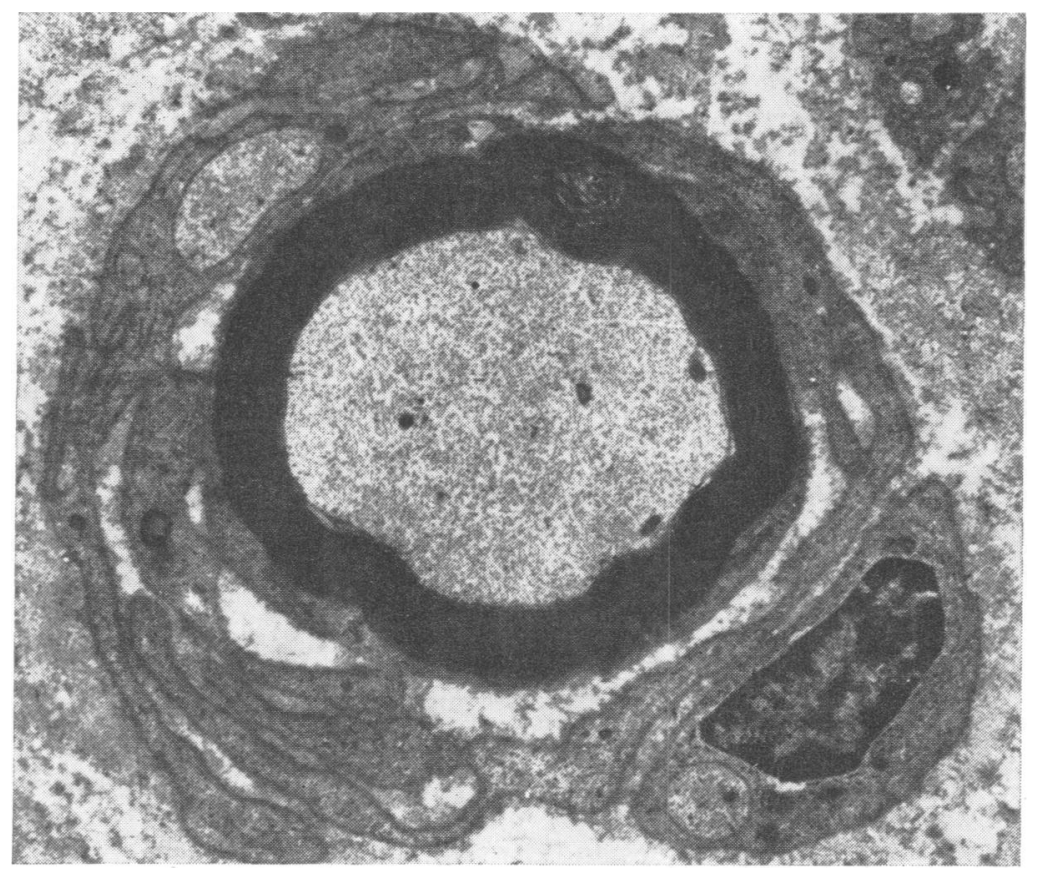

FIG. 4 Cytoplasm of the Schwann cell in biopsied sural nerve has many processes which concentrically surround a myelinated nerve, indicating onion bulb formation. $\times 6300$. 


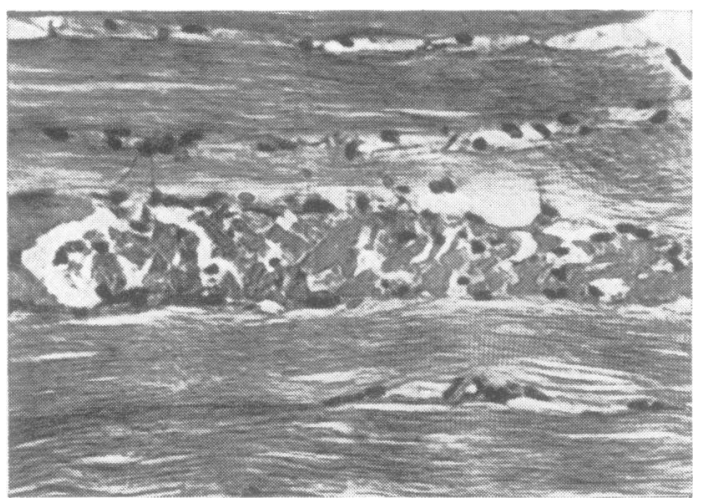

FIG. 5 Peroneus brevis muscle biopsy specimen. One muscle fibre shows floccular degeneration with myophagia. $H$ and $E, \times 260$.

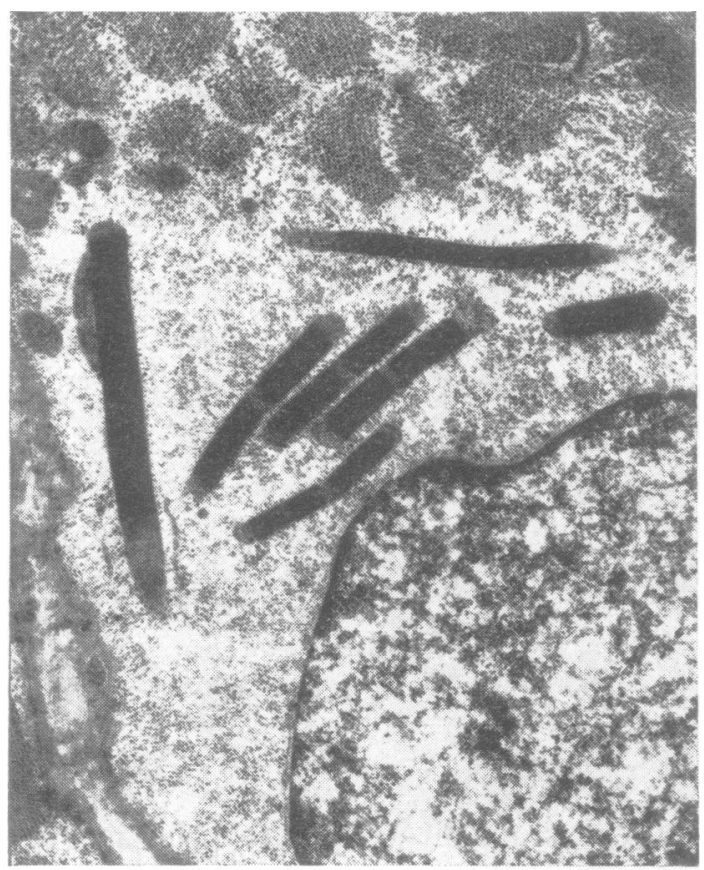

FIG. 6 Electron micrograph of peroneus brevis muscle. Mitochondria are elongated and contain a rectangular inclusion body. Glycogen granules are abundant among myofibrils and around the nucleus. $\times 9700$. microscope. The second segment of the nerve was fixed in $2.5 \%$ glutaraldehyde, postfixed in $1 \%$ osmic acid and embedded in epoxy resin. Semi-thin sections were stained with toluidine blue for analysis of the myelinated nerve fibre diameter. Ultra-thin sections were doubly stained with uranyl acetate and lead citrate for an electron microscopic study.

The specimen obtained from the left peroneus brevis muscle was also divided into two fragments. The first part was used for a light microscopic examination, stained with haematoxylin and eosin, phosphotungstic acid haematoxylin, van Gieson, Mallory-azan, periodic acid-Schiff, mucicarmine, toluidine blue, and alcian blue. The remainder of the muscle was fixed in $2.5 \%$ glutaraldehyde, postfixed in $1 \%$ osmic acid, and embedded in epoxy resin. Ultra-thin sections were stained with uranyl acetate and lead citrate and examined with an electron microscope.

\section{RESULTS}

The sural nerve of paraffin sections disclosed considerable demyelination, especially of large fibres, with mild proliferation of Schwann cells $\omega$ and mast cells. Collagen fibres were increased among the destroyed nerve fibres. Beneath the 음 perineurium, which was not thickened, smalk amounts of floccular materials were observed. 3 These were stained metachromatically witho toluidine blue, positively with alcian blue, and옹 negatively with periodic acid-Schiff. Theifo $\overrightarrow{0}$ quantity, however, was not more extensive than or in normal controls. There was no thickening of the vascular walls. Perivascular or interfascicular inflammatory cell infiltrates were not observed.

On teased specimens, segmental demyelination was apparent. In some fibres demyelination was restricted only to the region of Ranvier's node or Schmidt-Lantermann's cleft (Fig. 1).

Histograms of the fibre diameter of the myelinated nerve fibres by semi-thin sections stained with toluidine blue demonstrated a fair decrease of large fibres, no longer showing normal bimodal distribution (Figs 2, 3). A total fibre density of the myelinated nerves was 6133 fibres per $\mathrm{mm}^{2}$.

Electron microscopically, large myelinated fibres were relatively occasionally encountered. Naked axons of a large diameter were also frequently present. Myelin and axons of the remaining myelinated nerve fibres were well preserved in general. There were myelin figures, lipid bodies and dense bodies in the cytoplasm of the 
Schwann cells. At times cytoplasm of the Schwann cells had many processes concentrically surrounding the myelinated nerves, indicating onion bulb formation (Fig. 4). Unmyelinated fibres were unremarkable. Collagen fibres were proliferated in the interstices. Fibroblasts and mast cells were incidentally seen.

Most of the muscle fibres of the left peroneus brevis muscle were 40 to 60 microns in diameter. Thin fibres with a diameter of 10 to 20 microns were scattered individually among them. A few fibres showed hyaline degeneration or floccular degeneration with myophagia (Fig. 5). Subsarcolemmal nuclei were occasionally increased in number. There were conglomerations or chain-like formations of subsarcolemmal nuclei in some places. One possible attempt at regeneration was noted. A small number of lymphocytes were incidentally seen among the muscle fibres. Proliferation of perimysial connective tissue and replacement by fatty tissue were of mild degree. Floccular materials, metachromatically stained with toluidine blue and positively with alcian blue, were indistinct.

Electron microscopically, the myofibrils were disorganized and occasionally destroyed with accumulation of lipid bodies and vacuoles. Considerable numbers of mitochondria were elongated and contained rectangular inclusion bodies. Glycogen granules were abundant among myofibrils, around the nuclei and especially beneath the sarcoplasmic membranes (Fig. 6). Some subsarcolemmal nuclei were increased in electron density.

\section{DISCUSSION}

The patient described here was found to suffer from primary myxoedema confirmed by several thyroid function tests. While paraesthesiae in hands have been commonly described in cases with myxoedema, which might be due to carpal tunnel syndrome produced by myxoedematous tissue beneath the transverse carpal ligament (Murray and Simpson, 1958; Purnell et al., 1961), our patient showed objective sensory impairment with mild muscle weakness in the distal part of the four extremities, delayed sensory conduction times and decreased deep tendon reflexes. This predominantly sensory polyneuropathy was markedly reversed by thyroid replacement therapy alone. This would afford strong evidence of causal association between hypothyroidism and polyneuropathy in our patient, not simply a chance association.

In 1961 Nickel and associates examined microscopically the peripheral nerves in cases with myxoedema. They observed an oedematous infiltration of the endoneurium and perineurium by a substance which stained metachromatically with aqueous toluidine blue stain and basophilically with haematoxylin and eosin, with an interstitial fibrosis. There were focal degenerative changes of the myelin sheaths and axis cylinders. They were of the opinion that the mucinous infiltrates found in the peripheral nerves could interfere mechanically with the metabolic exchange of nutrients and catabolic products to and from the neurone, resulting in peripheral neuropathy. Furthermore, Dyck and Lambert (1970) studied histologically sural nerves from two patients with diffuse polyneuropathy associated with hypothyroidism. They demonstrated segmental demyelination and remyelination in teased fibre studies. Under the electron microscope, aggregates of glycogen granules, mitochondria, lipid droplets, and lamellar bodies were found in Schwann cell cytoplasm. In contrast with Nickel et al. (1961), however, they saw only a slight increase in mucoid substances in nerves from their patients. They assumed from their observation that the Schwann cell changes appeared to predominate despite evidence of axis cylinder disease as well.

In the present study we also were unable to find significant increase of mucinous deposits in the sural nerve. Instead, the specimens demonstrated widespread segmental demyelination. It was considered that the quantity of the mucinous deposition was too little to cause a segmental demyelination by compression alone in our patient. In addition, electron microscopically, we found not a few naked axons of a large diameter and onion bulb formations which are usually assumed to be evidence of repeated demyelination and remyelination. These findings suggest that a derangement of the metabolism of Schwann cells due to hypothyroidism had produced the systemic polyneuropathy in our case.

Disturbed metabolism in hypothyroidism is partly evident as a decrease of oxygenation, altered glucose utilization, and an increase of 
serum cholesterol, beta-lipoprotein, creatine phosphokinase, lactic dehydrogenase and aldolase values, etc., most of which were seen in our patient. One or other of these disturbances, or some other unknown disturbances, may be responsible for metabolic derangement of Schwann cells. Dyck and Lambert (1970) reported that significantly large quantities of glycogen granules were seen as aggregates and in diffuse form in the peripheral nerves of myxoedematous patients. They had no reasons to suggest that the increase in glycogen was itself responsible for the segmental demyelination which they saw. Apparently, the increased glycogen could be ascribed to an imbalance of synthesis and degradation of glycogen as a result of hypothyroidism.

Experimentally induced hypothyroid state is also known to cause insufficient myelination, and retardation in the development of the central nervous system. There may be the same effect on the myelin of the peripheral nervous system in hypothyroidism, although there seems to be no distinct evidence regarding the effect of thyroid deficiency on the peripheral nervous system.

Although deficiency of thyroid stimulating hormone has been proved to be responsible for producing neuropathy (Grabow et al., 1968), bioassay titre of thyroid stimulating hormone in our patient was satisfactory, revealing the case to be one of primary hypothyroidism.

It appears reasonable to believe, in view of the present results, that myxoedematous polyneuropathy may be an intrinsic polyneuropathy due to metabolic disorder of Schwann cells based on thyroid hormone insufficiency, not merely a compressive neuropathy due to deposition of mucinous substance.

Specific myopathy has also been described in association with myxoedema (Astrom et al., 1961; Fessel, 1968). In myxoedematous myopathy, prolonged contraction and relaxation, muscular hypertrophy, pseudomyotonic syndrome, and painful spasms are mentioned besides proximal weakness (Wilson and Walton, 1959). Rarely, a myasthenic state may occur. Muscular hypertrophy with weakness and slowness of muscular contraction and relaxation has been described in children suffering from sporadic cretinism, which is referred to as Kocher-Debré-Sémélaigne syndrome (Najjar and
Nachman, 1965). When a similar condition with painful spasms and pseudomyotonia occurs in adults it is termed Hoffmann's syndrome.

Morphological changes in the skeletal muscles in hypothyroidism have been repeatedly described (Salick et al., 1968). Light microscopic studies of affected skeletal muscles disclose mild structural alterations such as cloudiness, metachromasia, and basophilia of the sarcoplasm, variation in fibre size, increase and central invasion of subsarcolemmal nuclei, segmental degeneration, phagocytosis, and collection of lymphocytes. Furthermore, Pearce and Aziz (1969) described hypertrophy and vacuolation of muscle fibres which had not been reported pre- is viously. Notable changes found by electron microscopy consist of focal areas of myofibrillar disorganization, nuclear proliferation, and mitochondrial alterations. Norris and Panner (1966) found linear and paracrystalline inclusions in the sarcoplasm and the mitochondria.

There was no clinical evidence suggestive of $\dot{\omega}$ myopathy in our patient. The weakness was is located in the distal muscles, which might be $\vec{\circ}$ attributed to the coexisting neuropathy. Th\& elevation of serum creatine phosphokinase is mor probably attributable to a promotion of formos tion of this enzyme in the thyroxin deficien condition than to associated myopathy. Histo $\overrightarrow{0}$ logical findings, however, revealed apparent ov myopathic changes of mild degree in addition to denervation atrophy probably due to myxoedematous neuropathy. This fact may suggest the presence of subclinical hypothyroid myo- ڤ pathy in addition to manifest polyneuropathy in $\triangle$ this case. Furthermore, rectangular inclusions in the mitochondria may suggest that the disturbed metabolism in hypothyroidism affected not only the Schwann cells but also the skeletal muscles.

\section{REFERENCES}

Astrom, K.-E., Kugelberg, E., and Muller, R. (1961). Hypothyroid myopathy. Archives of Neurology, 5, 472-482. 'ं

Collins, R. D., Berends, F., and Bittenbender, J. B. (1970). 윽 Neurological manifestations of hypothyroidism. Pennsylvania Medicine, 73, 35-38.

Crevasse, L. E., and Logue, R. B. (1959). Peripheral neuropathy in myxedema. Annals of Internal Medicine, 50, 14331437.

Dyck, P. J., and Lambert, E. H. (1970). Polyneuropathy $\bar{N}$ associated with hypothyroidism. Journal of Neuropathology and Experimental Neurology, 29, 631-658.

Fessel, W. J. (1968). Myopathy of hypothyroidism. Annals of $\mathrm{N}$ the Rheumatic Diseases, 27, 590-596. 
Grabow, J. D., and Chou, S. M. (1968). Thyrotropin hormone deficiency. With a peripheral neuropathy. Archives of Neurology, 19, 284-291.

Murray, I. P. C., and Simpson, J. A. (1958). Acroparaesthesia in myxoedema. A clinical and electromyographic study. Lancet, 1, 1360-1363.

Najjar, S. S., and Nachman, H. S. (1965). The KocherDebré-Sémélaigne syndrome. Hypothyroidism with muscular 'hypertrophy'. Journal of Pediatrics, 66, 901-908.

Nickel, S. N., and Frame, B. (1958). Neurologic manifestations of myxedema. Neurology (Minneap.), 8, 511-517.

Nickel, S. N., Frame, B., Bebin, J., Tourtellotte, W. W., Parker, J. A., and Hughes, B. R. (1961). Myxedema neuropathy and myopathy. A clinical and pathologic study. Neurology (Minneap.), 11, 125-137.

Norris, F. H., Jr, and Panner, B. J. (1966). Hypothyroid myopathy. Clinical, electromyographical, and ultrastructural observations. Archives of Neurology, 14, 574-589.
Pearce, J., and Aziz, H. (1969). The neuromyopathy of hypothyroidism. Some new observations. Journal of the Neurological Sciences, 9, 243-253.

Purnell, D. C., Daly, D. D., and Lipscomb, P. R. (1961). Carpal-tunnel syndrome associated with myxedema. Archives of Internal Medicine, 108, 751-756.

Salick, A. I., Colachis, S. C., Jr, and Pearson, C. M. (1968). Myxedema myopathy: clinical, electrodiagnostic, and pathologic findings in advanced case. Archives of Physical Medicine and Rehabilitation, 49, 230-237.

Sanders, V. (1962). Neurologic manifestations of myxedema. New England Journal of Medicine, 266, 547-552, 599-603.

Watanakunakorn, C., Hodges, R. E., and Evans, T. C. (1965). Myxedema. A study of 400 cases. Archives of Internal Medicine, 116, 183-190.

Wilson, J., and Walton, J. N. (1959). Some muscular manifestations of hypothyroidism. Journal of Neurology, Neurosurgery, and Psychiatry, 22, 320-324. 